\title{
Brown dwarfs as Galactic chronometers
}

\author{
Adam J. Burgasser \\ Massachusetts Institute of Technology, Kavli Institute for Astrophysics and Space Research, \\ Building 37, Room 664B, 77 Massachusetts Avenue, Cambridge, MA 02139, USA \\ email: ajb@mit.edu
}

\begin{abstract}
Brown dwarfs are natural clocks, cooling and dimming over time due to insufficient core fusion. They are also numerous and present in nearly all Galactic environments, making them potentially useful chronometers for a variety of Galactic studies. For this potential to be realized, however, precise and accurate ages for individual sources are required, a prospect made difficult by the complex atmospheres and spectra of low-temperature brown dwarfs; degeneracy between mass, age and luminosity; and the lack of useful age trends in magnetic activity and rotation. In this contribution, I review five ways in which ages for brown dwarfs are uniquely determined, discuss their applicability and limitations, and give current empirical precisions.
\end{abstract}

Keywords. stars: binaries, stars: fundamental parameters, stars: kinematics, stars: late-type, stars: low-mass, brown dwarfs.

\section{Introduction}

Brown dwarfs are very low-mass stars whose masses $\left(\mathrm{M} \lesssim 0.075 \mathrm{M}_{\odot}\right)$ are insufficient to sustain the core hydrogen fusion reactions that balance radiative energy losses (Kumar 1963; Hayashi \& Nakano 1963). Supported from further gravitational contraction by electron degeneracy pressure, evolved brown dwarfs continually cool and dim over time as they radiate away their initial contraction energy, ultimately achieving photospheric conditions that can be similar to those of giant planets. The first examples of brown dwarfs were identified as recently as 1995 (Nakajima et al. 1995; Rebolo et al. 1995). Today, there are hundreds known in nearly all Galactic environments, identified largely in wide-field, red and near-infrared imaging surveys such as 2MASS, DENIS, SDSS and UKIDSS. The known population of brown dwarfs encompasses the late-type $\mathrm{M}\left(\mathrm{T}_{e f f} \approx\right.$ $2500-3500 \mathrm{~K}), \mathrm{L}\left(\mathrm{T}_{e f f} \approx 1400-2500 \mathrm{~K}\right)$ and $\mathrm{T}$ spectral classes $\left(\mathrm{T}_{e f f} \approx 600-1400 \mathrm{~K}\right.$; e.g., Vrba et al. 2004), while efforts are currently underway to find even colder members of the putative Y dwarf class (see review by Kirkpatrick 2005).

Because brown dwarfs cool over time, their spectral properties are inherently timedependent, making them potentially useful chronometers for Galactic studies (much like white dwarfs; see contributions by M. Salaris, J. Kalirai, S. Catalán and H. Richer). However, the primary observables of a brown dwarf-temperature, luminosity and spectral type - depend on both mass and age (and weakly on metallicity). This degeneracy complicates characterizations of individual sources and mixed populations. Unfortunately, traditional stellar age-dating methods do not appear to be applicable for brown dwarfs. Magnetic activity metrics, such as the frequency and strength of optical or X-ray nonthermal emission, appear to be largely age-invariant (e.g., Stelzer et al. 2006) and quiescent emission drops off precipitously in the early $\mathrm{L}$ dwarfs as cool photospheres are decoupled from field lines $\dagger$ (e.g., Mohanty et al. 2002; see contribution by A. West). Long-term angular momentum loss in brown dwarfs is far more muted than in stars,

$\dagger$ Interestingly, radio emission does not drop off for cooler brown dwarfs, and may even increase relative to bolometric flux, although there are currently few detections (e.g., Berger 2006). 
Table 1. Age-dating Methods for Brown Dwarfs.

\begin{tabular}{|c|c|c|c|c|c|}
\hline Technique & Applicable for & Pros & Cons & Precision & $\mid \begin{array}{l}\text { Examples } \\
\text { in the } \\
\text { Literature }\end{array}$ \\
\hline $\begin{array}{l}\text { Cluster } \\
\text { members \& } \\
\text { companions }\end{array}$ & $\begin{array}{l}\text { nearby clusters; } \\
\text { companions to } \\
\text { age-dated stars }\end{array}$ & $\begin{array}{l}\text { precise ages based on } \\
\text { stellar/cluster work; } \\
\text { calibration for other } \\
\text { techniques and } \\
\text { evolutionary models }\end{array}$ & $\left|\begin{array}{l}\text { generally restricted to } \\
\text { young clusters; wide } \\
\text { (resolvable) \& close } \\
(\mathrm{RV}) \text { companions rare; } \\
\text { must verify coevality/ } \\
\text { association; atmospheres } \\
\text { variable for } t \lesssim 10 \mathrm{Myr}\end{array}\right|$ & $\left|\begin{array}{c}\sim 10 \% \text { clusters } \\
\sim 50-100 \% \text { for } \\
\text { companions }\end{array}\right|$ & $1,2,3,4$ \\
\hline $\begin{array}{l}6708 \AA \text { Li I } \\
\text { absorption }\end{array}$ & $\left|\begin{array}{l}t \sim 10-200 \mathrm{Myr} ; \\
\text { resolved binaries; } \\
\text { individual field } \\
\text { sources with } \\
\mathrm{T}_{\text {eff }} \geq 1500 \mathrm{~K} \\
\text { and } t \lesssim 2 \mathrm{Gyr} \\
\text { (limits only) }\end{array}\right|$ & $\begin{array}{l}\text { consistent predictions } \\
\text { from different models; } \\
\text { straightforward test; } \\
\text { largely insensitive to } \\
\text { atmospheric properties }\end{array}$ & $\mid \begin{array}{l}\text { requires high sensitivity, } \\
\text { high resolution spectra; } \\
\text { low brightness region; } \\
\text { not useful for } \mathrm{T} \text { dwarfs } \\
\text { or for } t \lesssim 10 \mathrm{Myr} ; \\
\text { relies on accurate } \\
\text { evolutionary models }\end{array}$ & $\begin{array}{c}\sim 10 \% \text { for } \\
\text { young clusters; } \\
\text { upper /lower } \\
\text { limits for all } \\
\text { others }\end{array}$ & $5,6,7$ \\
\hline $\begin{array}{l}\text { Mass } \\
\text { standards }\end{array}$ & $\begin{array}{l}\text { astrometric/RV } \\
\text { binaries }\end{array}$ & $\begin{array}{l}\text { precise masses } \\
\text { yield precise ages; } \\
\text { weakly sensitive } \\
\text { to atmospheric } \\
\text { properties }\end{array}$ & $\begin{array}{l}\text { suitable systems are } \\
\text { rare; long-term } \\
\text { follow-up required; } \\
\text { relies on accurate } \\
\text { evolutionary models }\end{array}$ & $\sim 10-20 \%$ & $8,9,10,11$ \\
\hline $\begin{array}{l}\text { Surface } \\
\text { gravities }\end{array}$ & $\begin{array}{l}\text { any source with } \\
\text { a well-measured } \\
\text { spectrum }\end{array}$ & $\begin{array}{l}\text { applicable to } \\
\text { individual sources; } \\
\text { particularly useful } \\
\text { for T dwarfs }\end{array}$ & $\left|\begin{array}{l}\text { low precision; other } \\
\text { factors (e.g., metallicity) } \\
\text { complicate analysis; } \\
\text { relies on accurate } \\
\text { evolutionary and } \\
\text { atmospheric models }\end{array}\right|$ & $\sim 50-100 \%$ & $12,13,14$ \\
\hline Kinematics & $\begin{array}{l}\text { well-defined } \\
\text { groups or } \\
\text { populations }\end{array}$ & $\begin{array}{l}\text { useful statistical test } \\
\text { for various subclasses; } \\
\text { insensitive to } \\
\text { evolutionary or } \\
\text { atmospheric models }\end{array}$ & $\begin{array}{l}\text { very low precision; } \\
\text { large groups required } \\
\text { to beat statistical noise; } \\
\text { susceptible to } \\
\text { selection biases }\end{array}$ & $\sim 100-300 \%$ & $\mid 15,16,17$ \\
\hline
\end{tabular}

References: (1) Bouvier et al. (1998); (2) Luhman et al. (2003); (3) Geballe et al. (2001); (4) Burgasser (2007); (5) Stauffer et al. (1998); (6) Jeffries \& Oliveira (2005); (7) Liu \& Leggett (2005); (8) Zapatero Osorio et al. (2004); (9) Stassun et al. (2006); (10) Liu et al. (2008); (11) Dupuy et al. (2008); (12) Mohanty et al. (2004); (13) Burgasser et al. (2006a); (14) Saumon et al. (2007); (15) Schmidt et al. (2007); (16) Zapatero Osorio et al. (2007); (17) Faherty et al. (2008).

and there is no clear rotation-activity relation for L dwarfs (e.g., Reiners \& Basri 2008). Exploitation of the cooling properties of brown dwarfs is therefore a favorable approach for determining their ages.

In this contribution, I review five methods currently employed to age-date brown dwarfs and summarize their applicability, inherent limitations and current (typical) precisions. Table 1 provides a summary of the methods discussed in detail below.

\section{Cluster members and companions}

The most straightforward way to age-date an individual brown dwarf is to borrow from its environment, a tactic that is suitable for members of coeval clusters/associations and companions to age-dated stars. Brown dwarfs are well-sampled down to and below the deuterium fusing mass limit $\left(\mathrm{M} \lesssim 0.013 \mathrm{M}_{\odot}\right)$ in the youngest nearby clusters $(t \lesssim 5 \mathrm{Myr})$, as their luminosities are greater at early ages. Brown dwarfs have also been identified in somewhat older (10-50 Myr) "loose associations" in the vicinity of the Sun $(d \lesssim 50$ 100 pc; e.g., Kirkpatrick et al. 2008). For older and more distant clusters $(d \gtrsim 1 \mathrm{kpc})$, decreasing surface temperatures and compact radii exacerbate the sensitivity issues that plague low-mass stellar studies (see contribution by G. Piotto). There are as yet no 
known brown dwarfs in globular clusters, despite detection of the end of the stellar main sequence in systems such as NGC 6397 (Richer et al. 2008).

For brown dwarfs in young clusters, numerous studies have examined age-related trends in colors (e.g., Jameson et al. 2008), spectral characteristics (e.g., Allers et al. 2007), accretion timescales (e.g., Mohanty et al. 2005) and circum(sub)stellar disk evolution (e.g., Scholz et al. 2007). These have been coarsely quantified, and appear to be most useful at very young ages $(t \lesssim 10 \mathrm{Myr}$ ). Surface properties and luminosities are highly variable at these ages due to sensitivity to formation conditions (e.g., Baraffe et al. 2002), ongoing accretion, complex magnetic effects (e.g., Reiners et al. 2007) and age spreads within a cluster (see contribution by R. Jeffries). Hence, while brown dwarfs in clusters with ages spanning $~ 1-650 \mathrm{Myr}$ are now well-documented, with age uncertainties as good as $10 \%$ (for the LDB technique; see $\S 3$ ), useful predictive trends are probably limited to ages of $\gtrsim 10 \mathrm{Myr}$.

Known brown dwarf companions to main sequence stars now number a few dozen, spanning a wide range of separation, age and composition. Many of these systems are widely-separated so that their brown dwarf companions can be directly studied. Age uncertainties for companion brown dwarfs depend on stellar dating methods which are generally more uncertain (50-100\%; e.g., Liu et al. 2008) than cluster ages. Searches for substellar companions to more precisely age-dated white dwarfs (e.g. Day-Jones et al. 2008; Farihi et al. 2008) and subgiants (Pinfield et al. 2006) have so far met with limited success. Nevertheless, brown dwarf companions to age-dated stars serve as important benchmarks for calibrating other age-dating methods at late ages ( $\gtrsim 500 \mathrm{Myr})$ and are fundamental for testing evolutionary models (see contribution by T. Dupuy).

\section{3. $6708 \AA$ Li I Absorption}

Lithium is fused at a lower temperature than hydrogen $\left(2.5 \times 10^{6} \mathrm{~K}\right)$, resulting in a somewhat lower fusing mass limit $\left(0.065 \mathrm{M}_{\odot}\right.$; Bildsten et al. 1997). Because the interiors of low mass stars and brown dwarfs are fully convective at early ages, an object with a mass above this limit will fully deplete its initial reservoir of lithium within $\sim 200$ Myr. Hence, any system older than this which exhibits Li I absorption has a mass less than $0.065 \mathrm{M}_{\odot}$ and is therefore a brown dwarf (e.g., Rebolo et al. 1992). With a mass limit, one can use evolutionary models to determine an age limit.

In the age range $\sim 10-200 \mathrm{Myr}$, the degree of lithium depletion in low-mass stars and brown dwarfs is itself mass-dependent, occurring earlier in more massive stars which first achieve the necessary core temperatures. Hence, over this range the age of an individual source can be precisely constrained if its mass is known. A more practical approach, however, is to ascertain the age of a group of coeval low-mass objects based on which sources do or do not exhibit Li I absorption; this is the lithium depletion boundary (LDB) technique. Different evolutionary models yield remarkably similar predictions for the location of the LDB over a broad range of ages (Burke et al. 2004), and the boundary itself is readily identifiable in color-magnitude diagrams. As such, this technique has been used to age-date several nearby young clusters and associations (e.g., Stauffer et al. 1998; Barrado y Navascués et al. 1999; Jeffries \& Oliveira 2005; Mentuch et al. 2008). LDB studies have also provided independent confirmation of other cluster-dating methods such as isochrone fitting (Jeffries \& Oliveira 2005). A variant of the LDB technique for coeval binary systems has been proposed by Liu \& Leggett (2005), in which a system that exhibits Li I absorption in the secondary but not in the primary can have both lower and upper age limits assigned to it (note that the presence/absence of $\mathrm{Li} \mathrm{I}$ in both components simply sets a single upper/lower age limit). This technique requires 
resolved optical spectroscopy of both components and can be pursued only for a few (rare) wide low-mass pairs (e.g., Burgasser et al., in prep.) or using high spatial-resolution spectroscopy (e.g. Martín et al. 2006). No single brown dwarf pair straddling the LDB has yet been identified.

Despite its utility, the detection of LI I absorption in brown dwarf spectra has limitations. The $6708 \AA$ line lies in an relatively faint spectral region for cool L-type dwarfs, so sensitive spectral observations on large telescopes are typically required to detect (or convincingly rule out) this feature. For optically-brighter M-type brown dwarfs, highresolution observations are generally required to distinguish $\mathrm{Li}$ I absorption from overlapping molecular absorption features. Young brown dwarfs $(t \lesssim 50 \mathrm{Myr})$ with low surface gravities show weakened alkali line absorption (see $\S 5$ ), including Li I, making it again necessary to obtain sensitive, high-resolution observations (Kirkpatrick et al. 2008). For brown dwarfs cooler than $\sim 1500 \mathrm{~K}$ (i.e., the $\mathrm{T}$ dwarfs), lithium is chemically depleted in the photosphere through its conversion to $\mathrm{LiCl}, \mathrm{LiF}$ or $\mathrm{LiOH}$ (Lodders 1999). As such, practical age constraints using Li I can only be made for systems younger than $\sim 2$ Gyr.

\section{Mass standards}

One way of breaking the mass/age/luminosity degeneracy for an individual brown dwarf is to explicitly measure its mass. This is feasible for sufficiently tight brown dwarf binaries for which radial velocity $(\mathrm{RV})$ and/or astrometric orbits can be measured. Of the $\sim 100$ very low mass $\left(\mathrm{M}_{1}, \mathrm{M}_{2} \leqslant 0.1 \mathrm{M}_{\odot}\right)$ binary systems now known, only a handful have sufficiently short periods that large portions of their RV orbits (e.g., Joergens \& Müller 2007; Blake et al. 2008), astrometric orbits (e.g., Lane et al. 2001; Bouy et al. 2004; Liu et al. 2008; Dupuy et al. 2008), or both (Zapatero Osorio et al. 2004; Stassun et al. 2006) have been measured. With measurable total system masses or mass functions, individual masses can be estimated from relative photometry or directly determined from recoil motion in both components (e.g., Stassun et al. 2006). The individual masses and component luminosities can then be compared to evolutionary models to determine ages.

Liu et al. (2008) have suggested that such "mass standards" provide more precise constraints on the physical properties (including ages) of brown dwarfs as compared to "age standards", namely companions to main sequence stars. Orbital masses can currently be constrained to roughly $5-10 \%$ precision, translating into $10-20 \%$ uncertainties in ages based on evolutionary tracks (versus $50-100 \%$ for main-sequence stars). More importantly, brown dwarf binaries with mass measurements and independent age determinations - i.e., companions to age-dated stars and cluster members - can provide specific tests of the evolutionary models themselves. Further details are provided in the contribution by T. Dupuy.

\section{Surface gravity}

Only 10-20\% of brown dwarfs are found to be multiple (e.g., Burgasser et al. 2006b) and few of these are suitable for orbital mass measurements. A proxy for mass is surface gravity, which can be determined directly from a brown dwarf's spectrum. For $\mathrm{T}_{e f f} \lesssim$ $2500 \mathrm{~K}$ and ages greater than $\sim 50 \mathrm{Myr}$, evolutionary models predict that brown dwarf surface gravities $\left(g \propto M / R^{2}\right)$ are roughly proportional to mass due to near-constant radii (roughly equal to Jupiter's radius). Surface gravity is also proportional to photospheric pressure $\left(P_{p h} \propto g / \kappa_{R}\right.$, where $\kappa_{R}$ is the Rosseland mean opacity), which in turn influences the chemistry, line broadening and (in some cases) opacities of absorbing species in the 
photosphere. Hence, "gravity-sensitive" features in a brown dwarf's spectrum can be used to infer its mass and, through evolutionary models, its age.

Examples of gravity-sensitive features include the optical and near-infrared VO bands and alkali lines in late-type $\mathrm{M}$ and $\mathrm{L}$ dwarfs, all of which evolve considerably between field dwarfs $(\log g \approx 5 \mathrm{cgs})$, young cluster dwarfs $(\log g \approx 3-4)$ and giant stars $(\log g \approx 0$; e.g., Luhman 1999). Enhanced VO absorption and weakened alkali line absorption is a characteristic trait of young brown dwarf spectra (e.g., Gorlova et al. 2003; Allers et al. 2007). Alkali features in particular are useful for cooler brown dwarfs as VO condenses out of the photosphere. Quantitative analyses of these features have been used to distinguish

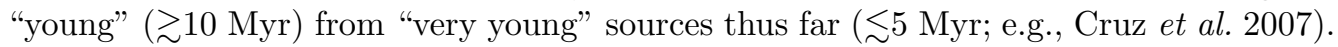
More robust metrics await larger and more fully-characterized samples.

Another important surface gravity diagnostic is collision-induced $\mathrm{H}_{2}$ absorption, a smooth opacity source spanning a broad swath of the infrared (e.g., Linsky 1969; Borysow et al. 1997). $\mathrm{H}_{2}$ absorption is weakened in the low-pressure atmospheres of young cluster brown dwarfs, resulting in reddened near-infrared spectral energy distributions and colors; in particular, a characteristic, triangular-shaped H-band (1.7 $\mu \mathrm{m})$ flux peak (e.g., Lucas et al. 2001; Kirkpatrick et al. 2006). The proximity of many young and reddened brown dwarfs $(<100 \mathrm{pc})$ rules out ISM absorption as a primary source for this reddening. Jameson et al. (2008) have exploited this trend by using a proper-motion selected sample of nearby young cluster candidate members to infer an age/color/luminosity relation for brown dwarfs younger than $0.7 \mathrm{Gyr}$, with a stated accuracy of \pm 0.2 dex in log(age), or about $60 \%$ fractional uncertainty. Kinematically older low-mass stars and brown dwarfs in the Galactic disk (e.g., Faherty et al. 2008) and halo populations (e.g., Burgasser et al. 2003) exhibit unusually blue near-infrared colors due to enhanced $\mathrm{H}_{2}$ absorption. However, differences in metallicities and condensate cloud properties can muddle surface gravity determinations in these sources by modulating the photospheric pressure through opacity effects (changing $\kappa_{R}$; e.g., Leggett et al. 2000; Looper et al. 2008).

The use of $\mathrm{H}_{2}$ absorption as a surface gravity indicator is particularly useful for $\mathrm{T}$ dwarfs, as $\mathrm{H}_{2}$ dominates the $K$-band $(2.1 \mu \mathrm{m})$ opacity and significantly influences nearinfrared colors (e.g., Burgasser et al. 2002; Knapp et al. 2004). Several groups now employ this feature to estimate the atmospheric properties of individual T dwarfs (e.g., Burgasser et al. 2006a; Warren et al. 2007; Burningham et al. 2008), typically through the use of spectral indices that separately sample surface gravity (e.g., the $K$-band) and temperature variations (e.g., $\mathrm{H}_{2} \mathrm{O}$ or $\mathrm{CH}_{4}$ bands). These indices are compared to atmospheric models calibrated by one or more benchmarks (e.g., a companion to a precisely age-dated star), and evolutionary models are used to determine individual masses and ages. Typical uncertainties of $\log g \sim 0.3$ dex translate into $50-100 \%$ uncertainties in age, comparable to uncertainties for main sequence stars. Again, variations in metallicity can mimic variations in surface gravity, although a third diagnostic such as luminosity can break this degeneracy (e.g., Burgasser 2007). As atmosphere models improve in fidelity, parameters are increasingly inferred from direct fits to spectral data, with comparable uncertainties (e.g., Saumon et al. 2007; Cushing et al. 2008).

\section{Kinematics}

When a sufficiently large enough sample of brown dwarfs is assembled, one can apply standard kinematic analyses, building from the assumption that gravitational perturbations lead to increased velocity dispersions with time (e.g., Spitzer \& Schwarzschild 1953; see contribution by B. Nordström). Velocity dispersions are typically characterized by a time-dependent power-law form, i.e., $\sigma \propto(1+t / \tau)^{\alpha}$ (e.g., Wielen 1977; Hänninen 
\& Flynn 2002). Other statistics, such as Galactic scale height, can also be tied to age through kinematic simulations (e.g., West et al. 2008) to calibrate secondary age diagnostics such as magnetic activity (see contribution by A. West).

Samples of very low mass stars and brown dwarfs have only recently become large enough that kinematic studies are feasible. The largest samples (over 800 sources) have been based on proper motion measurements (e.g., Schmidt et al. 2007; Casewell et al. 2008; Faherty et al. 2008). For field dwarfs, dispersion in tangential velocities for both magnitude- and volume-limited samples indicate a mean age in the range 2-8 Gyr, largely invariant with spectral type. This is consistent with the ages of more massive field stars and population synthesis models (e.g., Burgasser 2004). However, when field samples are broken down by color (Faherty et al. 2008) or presence of magnetic activity (Schmidt et al. 2007), distinct age groupings are inferred, indicating that both very old (i.e., thick disk or halo) and very young (i.e., thin disk or young association) brown dwarf populations coexist in the immediate vicinity of the Sun. Indeed, one of the major results from these studies is the identification of widely-dispersed brown dwarf members of nearby, young moving groups such as the Hyades (e.g., Bannister \& Jameson 2007).

With only two dimensions of motion measured, proper motion samples may produce biased dispersion measurements depending on the area of sky covered by a sample. Full $3 \mathrm{D}$ velocities require RV measurements which are more expensive and have thus far been obtained only for a small fraction of the known brown dwarf population (e.g., Basri \& Reiners 2006; Blake et al. 2007). A seminal study by Zapatero Osorio et al. (2007) of 21 nearby, late-type dwarfs with parallax, proper motion and RV measurements found considerably smaller 3D velocity dispersions for L and T dwarfs than GKM stars, suggesting that local brown dwarfs are young $(t \sim 0.5-4$ Gyr $)$. The discrepancy between this result and the proper motion studies may be attributable to small number statistics and/or contamination by young moving groups; $~ 40 \%$ of the brown dwarfs in the Zapatero Osorio et al. (2007) sample appear to be associated with the Hyades. Resolving this discrepancy requires larger RV samples, which has the side benefit of potentially uncovering RV variables that can be used as mass standards (e.g., Basri \& Reiners 2006).

\section{Improvements and future work}

With several methods for age-dating brown dwarfs over a broad range of phase space now available, opportunities to use these objects as chronometers for various Galactic studies look to be increasingly promising; e.g., age-dating planetary systems, examining cluster age spreads, testing Galactic disk dynamical heating models, and direct measures of the substellar mass function and birthrate in the field and other populations. However, there are areas where improvements in uncertainties are needed and basic assumptions tested. Surface gravity determinations in particular require better constraints, since these enable age-dating of individual sources. In the short term, improvements in spectral models can help in this endeavor; however, a sufficiently sampled grid of benchmark sources may obviate the need for models entirely. Benchmarks should increasingly arise from mass standards, for which age constraints are more precise; these additionally provide necessary tests of evolutionary models upon which most of the age-dating techniques hinge. Improved angular resolution and sensitivity with JWST and the next generation of large $(>25 \mathrm{~m})$ telescopes will increase resolved binary sample sizes by expanding the volume in which they can be found and monitored. These facilities will also aid searches for substellar cluster members in old open field and globular clusters and, perhaps more importantly, mass standards in these clusters to facilitate more stringent tests of evolutionary models (T. Dupuy, priv. comm.). Finally, a larger, more complete sample of 
brown dwarfs with precise RV measurements will both improve our statistical constraints on the age of the local brown dwarf population (and subpopulations) while additionally aiding in the search for mass standards.

The author thanks T. Dupuy, S. Leggett, M. Liu, \& A. West for helpful comments.

\section{References}

Allers, K. N., Jaffe, D. T., Luhman, K. L., et al. 2007, ApJ, 657, 511

Bannister, N. P. \& Jameson, R. F. 2007, MNRAS, 378, L24

Baraffe, I., Chabrier, G., Allard, F., \& Hauschildt, P. H. 2002, A\& $A, 382,563$

Barrado y Navascués, D., Stauffer, J. R., \& Patten, B. M. 1999, ApJL, 522, L53

Basri, G. \& Reiners, A. 2006, AJ, 132, 663

Berger, E. 2006, $A p J, 648,629$

Bildsten, L., Brown, E. F., Matzner, C. D., \& Ushomirsky, G. 1997, ApJ, 482, 442

Blake, C. H., Charbonneau, D., White, R. J., Marley, M. S., \& Saumon, D. 2007, ApJ, 666, 1198

Blake, C. H., Charbonneau, D., White, R. J., Torres, G., Marley, M. S., \& Saumon, D. 2008, ApJL, 678, L125

Borysow, A., Jorgensen, U. G., \& Zheng, C. 1997, A\&A, 324, 185

Bouvier, J., Stauffer, J. R., Martin, E. L., Barrado y Navascues, D., Wallace, B., \& Bejar, V. J. S. 1998, A\&SA, 336, 490

Bouy, H., Duchêne, G., Köhler, R., et al. 2004, A\&A, 423, 341

Burgasser, A. J. 2004, ApJS, 155, 191

-. 2007, ApJ, 658, 617

Burgasser, A. J., Burrows, A., \& Kirkpatrick, J. D. 2006a, ApJ, 639, 1095

Burgasser, A. J., Kirkpatrick, J. D., Brown, M. E., et al. 2002, ApJ, 564, 421

Burgasser, A. J., Kirkpatrick, J. D., Burrows, A., Liebert, J., Reid, I. N., Gizis, J. E., McGovern, M. R., Prato, L., \& McLean, I. S. 2003, ApJ, 592, 1186

Burgasser, A. J., Kirkpatrick, J. D., Cruz, K. L., Reid, I. N., Leggett, S. K., Liebert, J., Burrows, A., \& Brown, M. E. 2006b, ApJS, 166, 585

Burke, C. J., Pinsonneault, M. H., \& Sills, A. 2004, ApJ, 604, 272

Burningham, B., Pinfield, D. J., Leggett, S. K., et al. 2008, MNRAS, 1183

Casewell, S. L., Jameson, R. F., \& Burleigh, M. R. 2008, MNRAS, 390, 1517

Cruz, K. L., Reid, I. N., Kirkpatrick, J. D., Burgasser, A. J., Liebert, J., Solomon, A. R., Schmidt, S. J., Allen, P. R., Hawley, S. L., \& Covey, K. R. 2007, AJ, 133, 439

Cushing, M. C., Marley, M. S., Saumon, D., Kelly, B. C., Vacca, W. D., Rayner, J. T., Freedman, R. S., Lodders, K., \& Roellig, T. L. 2008, ApJ, 678, 1372

Day-Jones, A. C., Pinfield, D. J., Napiwotzki, R., Burningham, B., Jenkins, J. S., Jones, H. R. A., Folkes, S. L., Weights, D. J., \& Clarke, J. R. A. 2008, MNRAS, 388, 838

Dupuy, T. J., Liu, M. C., \& Ireland, M. J. 2008, ArXiv e-prints

Faherty, J. K., Burgasser, A. J., Cruz, K. L., Shara, M. M., Walter, F. M., \& Gelino, C. R. 2008, ArXiv e-prints

Farihi, J., Becklin, E. E., \& Zuckerman, B. 2008, ApJ, 681, 1470

Geballe, T. R., Saumon, D., Leggett, S. K., Knapp, G. R., Marley, M. S., \& Lodders, K. 2001, ApJ, 556, 373

Gorlova, N. I., Meyer, M. R., Rieke, G. H., \& Liebert, J. 2003, ApJ, 593, 1074

Hänninen, J. \& Flynn, C. 2002, MNRAS, 337, 731

Hayashi, C. \& Nakano, T. 1963, Progress of Theoretical Physics, 30, 460

Jameson, R. F., Lodieu, N., Casewell, S. L., Bannister, N. P., \& Dobbie, P. D. 2008, MNRAS, 385,1771

Jeffries, R. D. \& Oliveira, J. M. 2005, MNRAS, 358, 13

Joergens, V. \& Müller, A. 2007, ApJL, 666, L113

Kirkpatrick, J. D. 2005, ARAA, 43, 195 
Kirkpatrick, J. D., Barman, T. S., Burgasser, A. J., McGovern, M. R., McLean, I. S., Tinney, C. G., \& Lowrance, P. J. 2006, ApJ, 639, 1120

Kirkpatrick, J. D., Cruz, K. L., Barman, T. S., et al. 2008, ArXiv e-prints, 808

Knapp, G. R., Leggett, S. K., Fan, X., et al. 2004, AJ, 127, 3553

Kumar, S. S. 1963, ApJ, 137, 1121

Lane, B. F., Zapatero Osorio, M. R., Britton, M. C., Martín, E. L., \& Kulkarni, S. R. 2001, ApJ, 560, 390

Leggett, S. K., Allard, F., Dahn, C., Hauschildt, P. H., Kerr, T. H., \& Rayner, J. 2000, ApJ, 535,965

Linsky, J. L. 1969, ApJ, 156, 989

Liu, M. C., Dupuy, T. J., \& Ireland, M. J. 2008, ArXiv e-prints

Liu, M. C. \& Leggett, S. K. 2005, ApJ, 634, 616

Lodders, K. 1999, ApJ, 519, 793

Looper, D. L., Kirkpatrick, J. D., Cutri, R. M., et al. 2008, ApJ, 686, 528

Lucas, P. W., Roche, P. F., Allard, F., \& Hauschildt, P. H. 2001, MNRAS, 326, 695

Luhman, K. L. 1999, ApJ, 525, 466

Luhman, K. L., Stauffer, J. R., Muench, A. A., Rieke, G. H., Lada, E. A., Bouvier, J., \& Lada, C. J. 2003, ApJ, 593, 1093

Martín, E. L., Brandner, W., Bouy, H., Basri, G., Davis, J., Deshpande, R., \& Montgomery, M. M. 2006, $A \mathscr{E} A, 456,253$

Mentuch, E., Brandeker, A., van Kerkwijk, M. H., Jayawardhana, R., \& Hauschildt, P. H. 2008, ArXiv e-prints

Mohanty, S., Basri, G., Jayawardhana, R., Allard, F., Hauschildt, P., \& Ardila, D. 2004, ApJ, 609,854

Mohanty, S., Basri, G., Shu, F., Allard, F., \& Chabrier, G. 2002, ApJ, 571, 469

Mohanty, S., Jayawardhana, R., \& Basri, G. 2005, ApJ, 626, 498

Nakajima, T., Oppenheimer, B. R., Kulkarni, S. R., Golimowski, D. A., Matthews, K., \& Durrance, S. T. 1995, Nature, 378, 463

Pinfield, D. J., Jones, H. R. A., Lucas, P. W., Kendall, T. R., Folkes, S. L., Day-Jones, A. C., Chappelle, R. J., \& Steele, I. A. 2006, MNRAS, 368, 1281

Rebolo, R., Martin, E. L., \& Magazzu, A. 1992, ApJL, 389, L83

Rebolo, R., Zapatero-Osorio, M. R., \& Martin, E. L. 1995, Nature, 377, 129

Reiners, A. \& Basri, G. 2008, ApJ, 684, 1390

Reiners, A., Seifahrt, A., Stassun, K. G., Melo, C., \& Mathieu, R. D. 2007, ApJL, 671, L149

Richer, H. B., Dotter, A., Hurley, J., et al. 2008, AJ, 135, 2141

Saumon, D., Marley, M. S., Leggett, S. K., et al. 2007, ApJ, 656, 1136

Schmidt, S. J., Cruz, K. L., Bongiorno, B. J., Liebert, J., \& Reid, I. N. 2007, AJ, 133, 2258

Scholz, A., Jayawardhana, R., Wood, K., Meeus, G., Stelzer, B., Walker, C., \& O'Sullivan, M. 2007, ApJ, 660, 1517

Spitzer, L. J. \& Schwarzschild, M. 1953, ApJ, 118, 106

Stassun, K. G., Mathieu, R. D., \& Valenti, J. A. 2006, Nature, 440, 311

Stauffer, J. R., Schultz, G., \& Kirkpatrick, J. D. 1998, ApJL, 499, L199+

Stelzer, B., Micela, G., Flaccomio, E., Neuhäuser, R., \& Jayawardhana, R. 2006, A\&̋A, 448, 293

Vrba, F. J., Henden, A. A., Luginbuhl, C. B., et al. 2004, AJ, 127, 2948

Warren, S. J., Mortlock, D. J., Leggett, S. K., et al. 2007, MNRAS, 381, 1400

West, A. A., Hawley, S. L., Bochanski, J. J., Covey, K. R., Reid, I. N., Dhital, S., Hilton, E. J., \& Masuda, M. 2008, $A J, 135,785$

Wielen, R. 1977, A\&\&A, 60, 263

Zapatero Osorio, M. R., Lane, B. F., Pavlenko, Y., Martín, E. L., Britton, M., \& Kulkarni, S. R. 2004, ApJ, 615, 958

Zapatero Osorio, M. R., Martín, E. L., Béjar, V. J. S., Bouy, H., Deshpande, R., \& Wainscoat, R. J. 2007, ApJ, 666, 1205 


\section{Discussion}

J. Melbourne: I was wondering how the brown dwarfs fit into the initial mass function?

A. Burgasser: There has been considerable work on this in young clusters, for example work by Luhman and Hillenbrand, and they find the IMF is definitely declining in the brown dwarf regime, likely peaking above $0.1 M_{\odot}$ (the Hyades may be somewhat different, however). In the field it is more difficult as one must invert the luminosity junction statiscally (again, part of the mass/ age/temperature degeneracy), but results there still indicate a decline (see question by Leggett). I would estimate that brown dwarfs are roughly equal in number to stars within a factor of a few.

S. LegGetT: Recent UKIDSS results (Pinfield, Chin) imply the MF is flat or declining at very low masses. Your thoughts?

A. Burgasser: The UKIDSS result is intriquing as it disagrees strongly with the 2MASS results. I worry a little bit about selection effects this early in the UKIDSS survey. It would be very useful to have an independent measure, for example from SDSS.

H. Richer: Can you comment on the use of the gap between the end of the hydrogenburning main sequence and brown dwarfs as a chronometer? We might see such a feature in 47 Tuc where we have a large program using WFC3 on HST coming up.

A. Burgasser: Detecting such a gap would be very interesting primarily from its constraint on the physics of "low" temperature $\mathrm{H}$ fusion in the cores of these objects. The evolutionary models show their largest differences near the H-burning mass limit, and in that sense ages based around that limit may be less reliable. However, this would be one of those important empirical checks on the models to increase our trust in their use.

M. LIU: 1) Regarding the discrepancy in the typical ages of field objects from the ZapteroOsorio and Faherty results, how much do selection effect of the two samples matter? 2) How reliable are the cluster/group memberships derived from proper motions (above)?

A. Burgasser: 1) I would say selction bias is very possible in the Zapetero-Osorio results given the small size of their otherwise well-characterized sample. The Faherty study specifically looked at a large sample limited to $20 \mathrm{pc}$; volume limited, even if not volume complete. There is of course a chance that our Sun lies in a special region surrounded by young low-mass stars in general, but that would be surprising. 2) The Jameson study has some problems with the older clusters where candidates are exclusively proper-motion selected. It would be very useful to confirm those sources spectroscopically.

J. StAuffer: If brown dwarfs do not spin down, then what you see is their birth angular momentum distribution (modulo how contraction or age is modifying this). Can't you use this information to then inform models of brown dwarf formation?

A. Burgasser: Possibly, although most models focus on much earlier times, well before BDs have fully contracted. An additional problem is actually measuring rotation periods, which is proving challenging as these objects are less variable than desired (largely due to lack of spots). But I think the large rotation velocities may have implications on the frequency of disks or close binaries among brown dwarfs. 


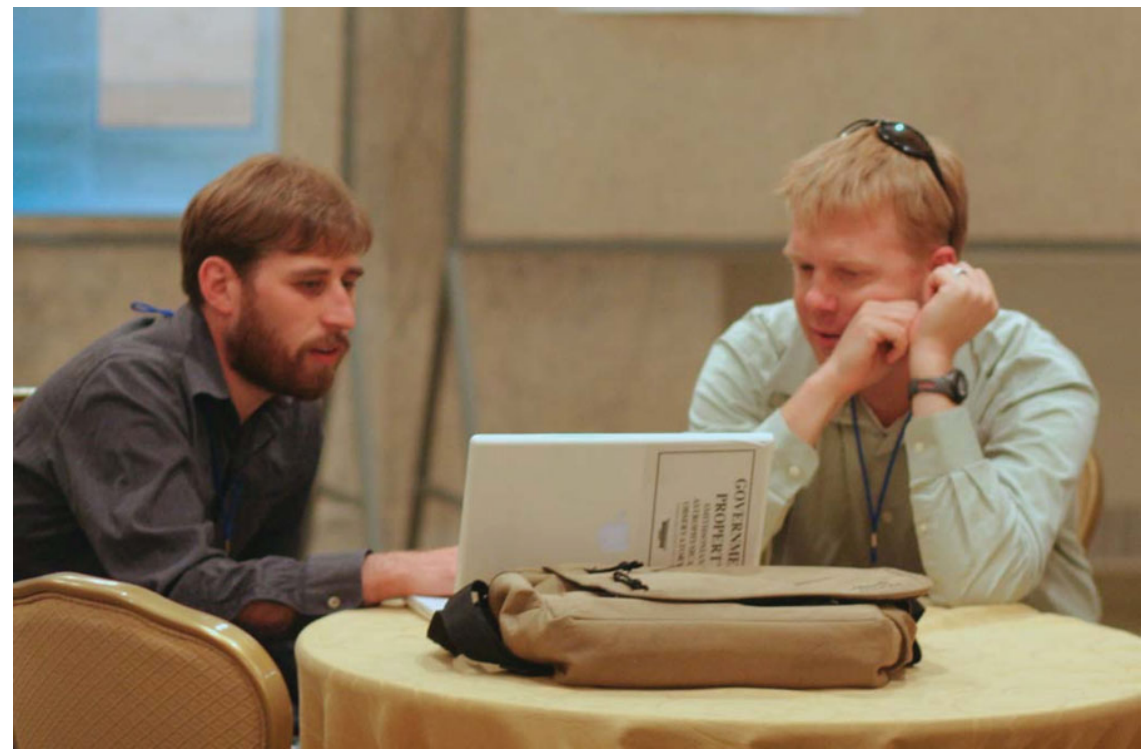

Kevin Covey and Andrew West

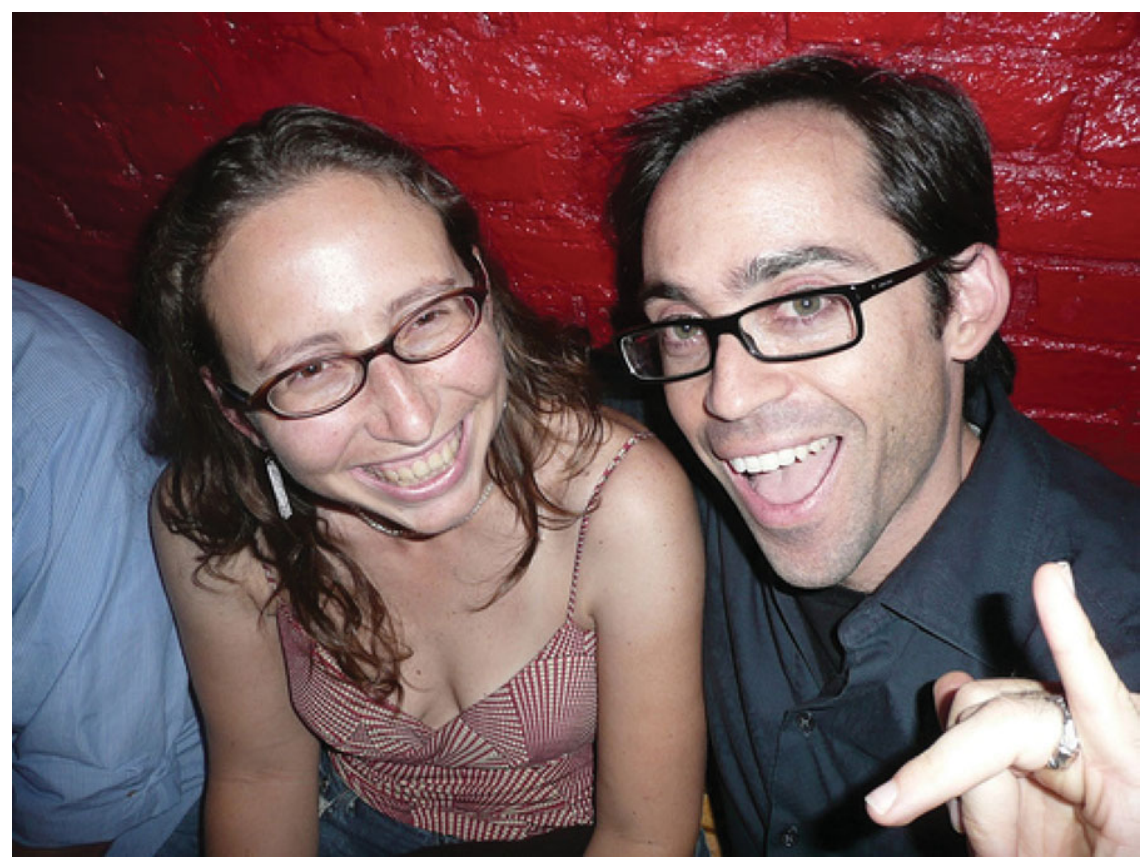

Kelle Cruz and Adam Burgasser 